\title{
Economics of resynchronization strategies including chemical tests to identify nonpregnant cows
}

\author{
J. O. Giordano, P. M. Fricke, and V. E. Cabrera ${ }^{1}$ \\ Department of Dairy Science, University of Wisconsin, Madison 53706
}

\section{ABSTRACT}

Our objectives were to assess (1) the economic value of decreasing the interval between timed artificial insemination (TAI) services when using a pregnancy test that allows earlier identification of nonpregnant cows; and (2) the effect of pregnancy loss and inaccuracy of a chemical test $(\mathrm{CT})$ on the economic value of a pregnancy test for dairy farms. Simulation experiments were performed using a spreadsheet-based decision support tool. In experiment 1, we assessed the effect of changing the interbreeding interval (IBI) for cows receiving TAI on the value of reproductive programs by simulating a 1,000-cow dairy herd using a combination of detection of estrus (30 to $80 \%$ of cows detected in estrus) and TAI. The IBI was incremented by $7 \mathrm{~d}$ from 28 to $56 \mathrm{~d}$ to reflect intervals either observed (35 to $56 \mathrm{~d}$ ) or potentially observed ( $28 \mathrm{~d}$ ) in dairy operations. In experiment 2 , we evaluated the effect of accuracy of the CT and additional pregnancy loss due to earlier testing on the value of reproductive programs. The first scenario compared the use of a CT $31 \pm 3 \mathrm{~d}$ after a previous AI with rectal palpation (RP) $39 \pm 3 \mathrm{~d}$ after AI. The second scenario used a CT $24 \pm 3 \mathrm{~d}$ after AI or transrectal ultrasound (TU) $32 \mathrm{~d}$ after AI. Parameters evaluated included sensitivity (Se), specificity (Sp), questionable diagnosis (Qd), cost of the CT, and expected pregnancy loss. Sensitivity analysis was performed for all possible combinations of parameter values to determine their relative importance on the value of the CT. In experiment 1, programs with a shorter IBI had greater economic net returns at all levels of detection of estrus, and use of chemical tests available on the market today might be beneficial compared with RP. In experiment 2 , the economic value of programs using a CT could be either greater or less than that of RP and TU, depending on the value for each of the parameters related to the CT evaluated. The value of the program using the CT was affected (in order) by (1) Se, (2) Sp, (3) pregnancy loss, (4) proportion of $\mathrm{Qd}$, (5) percentage of

Received May 8, 2012.

Accepted November 1, 2012.

${ }^{1}$ Corresponding author: vcabrera@wisc.edu cows AI in estrus, and (6) cost of CT. A change of $1 \%$ in the Se of the CT was 1.8 times more important than a similar change in Sp or pregnancy loss, and 13.7, 55.0, and 305.8 times more important than similar changes in Qd, cows inseminated in estrus, and cost of CT. We conclude that the major effect of using a $\mathrm{CT}$ is the potential of decreasing the IBI. Moreover, inaccuracy of the $\mathrm{CT}$ and additional pregnancy loss due to earlier testing resulted in smaller economic differences than when using RP or TU 8 d later.

Key words: simulation, modeling, reproductive program, pregnancy diagnosis

\section{INTRODUCTION}

Coupling resynchronization of ovulation programs with practical and cost-effective methods to identify nonpregnant cows as early as possible after a previous AI increases the AI service rate in a dairy herd by decreasing the interval between inseminations. The economic value of any test, however, will depend largely on the accuracy of the test to identify nonpregnant and pregnant cows correctly, as well as the cost of the test (Oltenacu et al., 1990; Galligan et al., 2009; Ferguson and Galligan, 2011). Therefore, diagnostic tests should have high sensitivity and specificity and generate the fewest possible questionable diagnoses.

Besides the traditional methods for nonpregnancy and pregnancy diagnosis in cattle (rectal palpation and transrectal ultrasound), new chemical methods for nonpregnancy diagnosis have been developed and are now commercially available. Assessment of pregnancy status through the detection of placental pregnancyassociated glycoproteins (PAG; Sasser et al., 1986; Zoli et al., 1992; Green et al., 2005) released into the maternal bloodstream is a reliable method to determine the pregnancy status of dairy cattle and can be incorporated into already established reproductive management schemes (Silva et al., 2007, 2009; Romano and Larson, 2010). Commercial PAG tests allow for determination of pregnancy in lactating dairy cows with good accuracy as early as 27 to 28 d after AI (Silva et al., 2007, 2009; Romano and Larson, 2010). As a consequence, one of the major advantages of a chemical test $(\mathbf{C T})$ is the 
potential for earlier identification of nonpregnant cows after an insemination, thereby allowing the implementation of aggressive resynchronization protocols that result in a short interbreeding interval (IBI).

Performing an early pregnancy test after AI may be highly beneficial because shortening the IBI improves reproductive performance (Giordano et al., 2011). However, like any other diagnostic method, CT may have low accuracy if used too early after AI. Another factor that affects the performance of an early CT is the occurrence of pregnancy loss, which is detrimental to the overall reproductive performance of the herd. Naturally occurring pregnancy losses in lactating dairy cows are more likely during the early stages of pregnancy and tend to decrease as gestation progresses (Vasconcelos et al., 1997; Santos et al., 2004; Giordano et al., 2012b). As a result, the earlier a pregnancy test is performed, the greater the number of pregnancies that will be lost until the next pregnancy reconfirmation or calving. Therefore, a tradeoff exists between the time gained with earlier pregnancy tests and the effect of pregnancy loss, because cows losing their pregnancy should be identified and resubmitted for AI as soon as possible after the loss.

Previous analyses have reported varying degrees of economic difference between the use of a CT, transrectal ultrasound (TU), or rectal palpation (RP) to detect pregnancy in dairy cattle (Oltenacu et al., 1990; Galligan et al., 2009; Ferguson and Galligan, 2011). These previous studies have relied on cost/benefit and partial budgeting analysis combined with some type of decision-tree framework (Oltenacu et al., 1990; Galligan et al., 2009; Ferguson and Galligan, 2011). Such approaches seem suitable because they can include the sensitivity (and the positive predictive value of the test), the specificity (and the negative predictive value of the test), and the probability of pregnancy loss within a framework to calculate the economic impact of these factors. Nonetheless, to measure the effect of a pregnancy test on the overall reproductive efficiency of a dairy herd, we chose to use a more comprehensive approach that allows for the dynamics of the whole lactating herd to be followed through repetitive breeding cycles and complete lactations. Integrating the pregnancy testing method within the reproductive program, rather than applying it as an isolated event, may better reflect its impact on reproductive dynamics, generating results of greater utility for producers and dairy industry consultants.

The objectives of the present study were to (1) assess the economic value of decreasing the interval between inseminations when using a nonpregnancy test that allows earlier identification of nonpregnant cows; and (2) assess the effects of pregnancy loss and inaccuracy of the $\mathrm{CT}$ on its value as a method for earlier nonpregnancy diagnosis in lactating dairy cows. Two simulation experiments were performed using a spreadsheet-based decision support tool (UW-DairyRepro\$; Giordano et al., 2011) that simulates the reproductive, productive, and economic dynamics of a dairy herd. Our main hypothesis was that the economic advantage of performing an earlier pregnancy test is the reduction in the interval between 2 successive inseminations. Moreover, we hypothesized that the improved reproductive efficiency due to a shorter IBI would offset any potential additional losses incurred due to inaccuracy of the earlier nonpregnancy diagnosis with a CT as well as the additional cost of performing the test.

\section{MATERIALS AND METHODS}

\section{Experiment 1: Model Description}

To assess the effect of different IBI on the reproductive dynamics and economics of a dairy operation, a 1,000-cow commercial dairy herd was simulated using the UW-DairyRepro\$ decision support tool (Giordano et al., 2011). Briefly, based on multiple herd descriptive and reproductive input parameters, the model simulates the reproductive dynamics of a herd of lactating dairy cows. The model estimates sequentially, as finite Markov chains, the percentage of cows eligible for insemination after the end of the voluntary waiting period (VWP), the proportion of cows receiving AI, the percentage of cows that become pregnant, and the percentage of cows that fail to become pregnant after each AI service. The first state in the Markov chain process is represented by the nonpregnant cows, which could move to the next state of being inseminated, becoming pregnant, being culled from the herd, or dying, following transition probabilities determined by the reproductive program under consideration. The model simultaneously calculates a future expected monetary value (based on milk income over feed cost, cost of nonreproductive culling and mortality, cost of reproductive culling, value of newborn calves, and the cost of reproductive programs) for cows becoming pregnant at different DIM between the end of the VWP and a predefined cutoff DIM for insemination, as well as for cows that remain nonpregnant by the end of the breeding period. The expected monetary value (indicates the income generated by cows) calculated for specific DIM for pregnant and nonpregnant cows is then multiplied by the percentage of cows in each reproductive status to estimate the net present value (NPV; $\$ /$ cow per year) of a reproductive program (Giordano et al., 2011). The discount rate entered as input for the calculation of NPV was set at $5.0 \%$. 


\section{Experiment 1: Comparison of Reproductive Programs}

The baseline reproductive management program consisted of a combination of estrus detection (ED) and timed AI (TAI) after synchronization of ovulation for first postpartum AI using a Presynch-Ovsynch protocol (Moreira et al., 2001) and the Ovsynch protocol (Pursley et al., 1995) for second and subsequent TAI services. Between the time of the second $\mathrm{PGF}_{2 \alpha}$ injection of Presynch coincident with the end of the VWP set at 50 DIM and the first GnRH injection (62 DIM) of the Ovsynch protocol for first TAI, cows detected in estrus were assumed to be inseminated. For second and subsequent AI services, AI after detection of estrus (AIED) was also performed between TAI services (Figure $1 \mathrm{~A}$ and B). Different programs were simulated by increasing the proportion of cows inseminated after ED from 30 to $80 \%$ in increments of 10 percentage points for all AI services. The expected CR for all AIED was set at $35 \%$ (Table 1). Under most circumstances, when ED is added before or between TAI services, a lower CR is observed for those cows inseminated to TAI because the population of cows reaching that point is different than when no estrus detection is added (Chebel et al., 2010; Chebel and Santos, 2010; Gumen et al., 2012). Therefore, for each 10-percentage-point increment in AIED before first-service TAI, the initial CR of $40 \%$ after TAI services decreased by 2 percentage points (Table 1). Additionally, the CR of second and subsequent TAI services decreased by 2 percentage points when the proportion of cows AIED was between 60 and $80 \%$ (Table 1 ). Once the baseline reproductive programs were defined, the IBI for cows receiving a TAI service was incremented by $7 \mathrm{~d}$ from 28 to $56 \mathrm{~d}$ to reflect intervals either observed (35 to $56 \mathrm{~d}$ ) or potentially observed $(28 \mathrm{~d})$ in commercial dairy operations when using resynchronization of ovulation for second and subsequent AI services. In all cases, the Ovsynch protocol for resynchronization was initiated before the nonpregnancy diagnosis, which was performed at the time of the $\mathrm{PGF}_{2 \alpha}$ injection of the Ovsynch protocol when RP and TU were used (Figure $1 \mathrm{~A}$ and $1 \mathrm{~B}$ ). In contrast, when a CT was used for nonpregnancy diagnosis, blood samples were assumed to be collected $1 \mathrm{~d}$ before the $\mathrm{PGF}_{2 \alpha}$ injection of the Ovsynch protocol to account for the time required to obtain the test results.

The same conception rate (CR) was used for all resynchronized TAI services regardless of the different IBI (Figure 1A and 1B). This approach was taken to simplify calculations and to better interpret results despite some potential differences that may be observed. Although lower fertility was observed when Ovsynch was initiated 19 d after a previous AI (Fricke et al., 2003), the model allows for the possibility that newer resynchronization strategies could be developed to improve conception rates at shorter IBI. Economic parameters (i.e., milk price, cull cow cost, heifer replacement cost, female calf cost, feed cost) used as inputs in the model were compiled from the Wisconsin Calculated Milk Cost of Production reported by the Center for Dairy Profitability at the University of Wisconsin-Madison for the months of April 2010 to April 2011 (http:// cdp.wisc.edu/MilkProductionCosts.htm). Additional input parameters (i.e., milk production curves, culling, mortality rate) were compiled from a DairyComp305 (Valley Agricultural Software, Tulare, CA) back-up file from a commercial 1,000-cow dairy operation in Wisconsin.

Considering the minimum number of days after AI that each of the nonpregnancy tests can be performed accurately and the integration of the test within a resynchronization program initiated weekly, the IBI observed under field conditions and included in this simulation model were 42 to $56 \mathrm{~d}$ for RP, 35 to $56 \mathrm{~d}$ for TU, and 28 to $56 \mathrm{~d}$ for a CT. Even though current commercial CT do not allow for nonpregnancy diagnosis at $21 \mathrm{~d}$ after AI (the earliest timing included in these simulation experiments), a program with such timing for the pregnancy test was included to explore the potential economic benefits and pitfalls of a very early nonpregnancy diagnosis. Nevertheless, recent research reports have demonstrated that chemical tests based on detection of PAG can accurately detect pregnancy as early as 25 d after AI (Green et al., 2009, 2011; Branen et al., 2011). In addition, new diagnostic tests based on the detection of IFN- $\tau$ stimulated genes in blood leukocytes (Gifford et al., 2007; Stevenson et al., 2007; Oliveira et al., 2008) are being developed for nonpregnancy diagnosis for use as early as 18 to $20 \mathrm{~d}$ after AI (Green et al., 2010) and may be available to the dairy industry in the future.

\section{Experiment 2: Economic Impact of the CT Performance for Nonpregnancy Diagnosis}

For this experiment, the UW-DairyRepro\$ decision support tool (Giordano et al., 2011) was modified to study the economic impact of performing nonpregnancy tests at different times after AI. Selected reproductive programs described in experiment 1 (Table 1) with specific modifications were used to test plausible scenarios of pregnancy testing, as defined in Table 2. Two sets of programs were simulated to assess the impact of shortening the IBI by $7 \mathrm{~d}$ due to an earlier nonpregnancy diagnosis using a CT. In all cases, the basic programs were similar to those described for experiment 1, combining ED for AI services with 


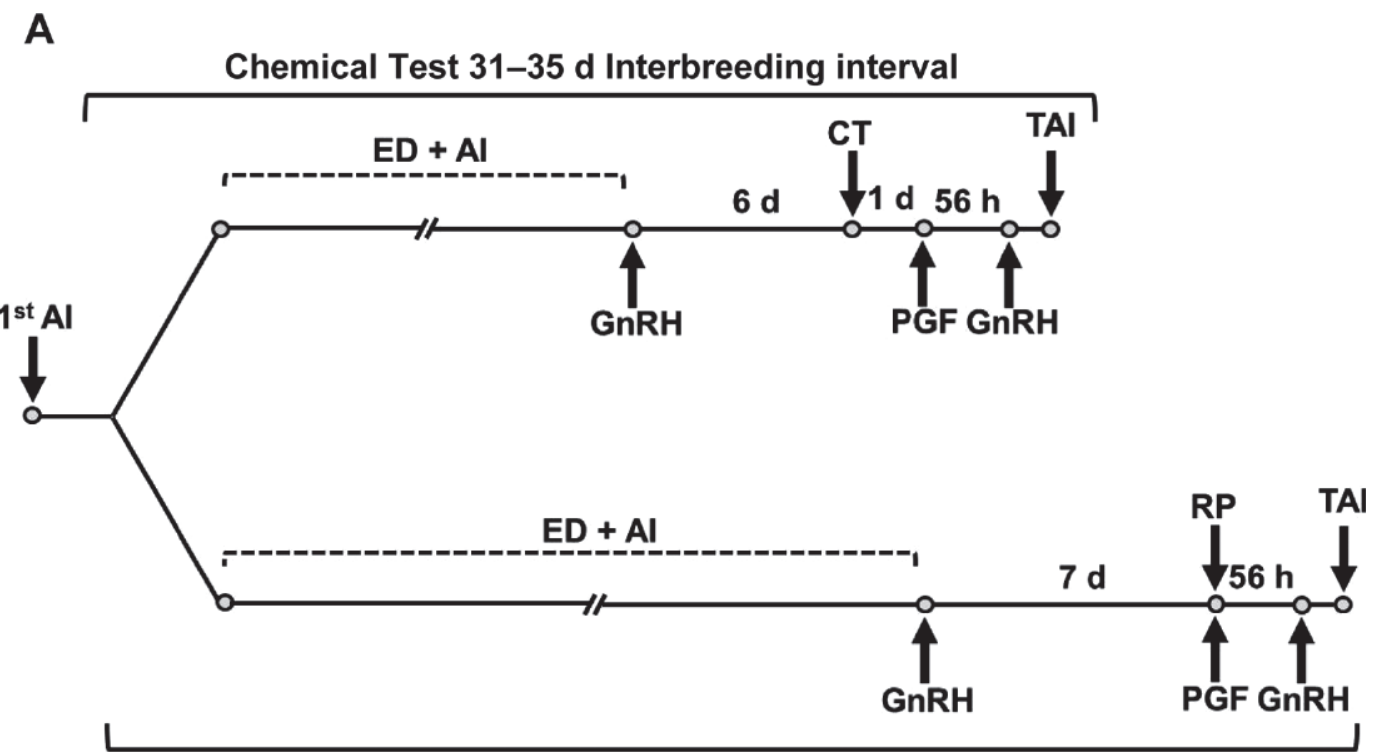

Rectal Palpation 39-42 d Interbreeding interval

B

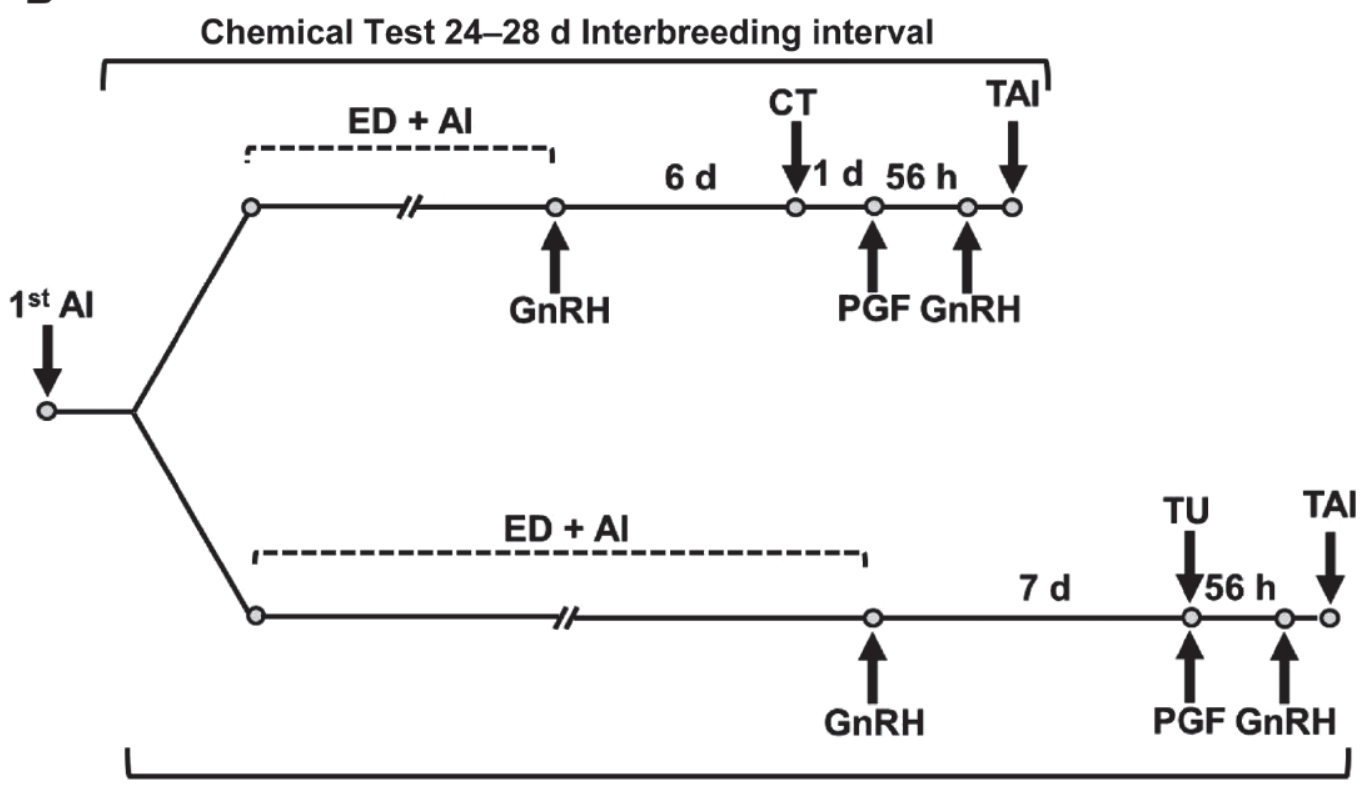

Transrectal Ultrasound 32-35 d Interbreeding interval

Figure 1. Reproductive programs simulated in experiment 2. In both cases, first AI service was performed after detection of estrus (ED) or after a Presynch-Ovsynch protocol. The first scenario (A) simulated consisted of 2 programs: the early testing program (CT31) used a chemical test (CT) $31 \pm 3$ d after a previous AI, whereas the late testing program (RP39) used rectal palpation (RP) $39 \pm 3$ d after AI. For CT31, cows not inseminated after detection of estrus (AIED) initiated an Ovsynch protocol $25 \pm 3 \mathrm{~d}$ after AI by receiving a GnRH injection. Cows diagnosed nonpregnant by the $\mathrm{CT}$ received the $\mathrm{PGF}_{2 \alpha}$ injection of Ovsynch $32 \pm 3 \mathrm{~d}$ after AI. For RP39cows, cows not AIED initiated an Ovsynch protocol $32 \pm 3 \mathrm{~d}$ after AI by receiving a GnRH injection. Cows diagnosed nonpregnant using RP received the PGF ${ }_{2 \alpha}$ injection of the Ovsynch protocol $39 \pm 3 \mathrm{~d}$ after AI. Thereafter, nonpregnant cows from both groups completed the Ovsynch protocol and received their next TAI. The resulting average interbreeding interval (IBI) was 35 and $42 \mathrm{~d}$ for CT31 and RP39, respectively. For the second comparison (B), the early testing program used a CT $24 \pm 3 \mathrm{~d}$ after a previous AI (CT24), whereas the late testing program used transrectal ultrasound (TU) $32 \pm 3 \mathrm{~d}$ after AI (TU32). For CT24, cows not AIED initiated the Ovsynch protocol $18 \pm 3$ d after AI by receiving a GnRH injection. Cows diagnosed nonpregnant by the $\mathrm{CT}$ received the $\mathrm{PGF}_{2 \alpha}$ injection of the Ovsynch protocol $25 \pm 3 \mathrm{~d}$ after AI. For TU32, cows not AIED initiated the Ovsynch protocol 25 $\pm 3 \mathrm{~d}$ after $\mathrm{AI}$ by receiving a GnRH injection. Cows diagnosed nonpregnant by TU received the $\mathrm{PGF}_{2 \alpha}$ injection of Ovsynch $32 \pm 3 \mathrm{~d}$ after $\mathrm{AI}$. Thereafter, nonpregnant cows from both groups completed the Ovsynch protocol and received their next TAI. The resulting average IBI was then 28 and $35 \mathrm{~d}$ for CT24 and TU32, respectively. In all cases, the programs selected were those in which $50 \%$ of cows were AIED. 
Table 1. Reproductive performance parameter inputs for the reproductive management programs simulated in experiment $1^{1}$

\begin{tabular}{|c|c|c|c|c|c|c|c|}
\hline Program & $\begin{array}{l}\text { Interbreeding } \\
\text { interval } \\
\text { (range, d) }\end{array}$ & \multicolumn{3}{|c|}{ First AI } & \multicolumn{3}{|c|}{ Second and subsequent AI } \\
\hline Presynch-Ovsynch and Ovsynch & $28-56$ & 40 & 35 & 38 & 40 & 35 & 30 \\
\hline Presynch-Ovsynch and Ovsynch & $28-56$ & 50 & 35 & 36 & 50 & 35 & 30 \\
\hline Presynch-Ovsynch and Ovsynch & $28-56$ & 60 & 35 & 34 & 60 & 35 & 28 \\
\hline
\end{tabular}

${ }^{1} \mathrm{AIED}=\mathrm{AI}$ after detection of estrus; $\mathrm{CR} \mathrm{ED}=$ conception rate of cows AI after detection of estrus; TAI $=$ timed AI.

the Presynch-Ovsynch and Ovsynch protocols for synchronization of ovulation and TAI in which $50 \%$ of the cows were inseminated after detection of estrus. The first simulation scenario consisted of 2 programs: the early testing program (CT31) used a CT at $31 \pm 3 \mathrm{~d}$ after a previous AI, whereas the late testing program (RP39) used RP at $39 \pm 3 \mathrm{~d}$ after AI (Figure 1A). For CT31, cows initiated the Ovsynch protocol $25 \pm$ $3 \mathrm{~d}$ after $\mathrm{AI}$ by receiving a $\mathrm{GnRH}$ injection, and cows diagnosed nonpregnant by the $\mathrm{CT}$ received the $\mathrm{PGF}_{2 \alpha}$ injection of the Ovsynch protocol $32 \pm 3 \mathrm{~d}$ after AI. In contrast, for RP39, cows initiated the Ovsynch protocol $32 \pm 3 \mathrm{~d}$ after AI by receiving a GnRH injection, and cows diagnosed nonpregnant by $\mathrm{RP}$ received the $\mathrm{PGF}_{2 \alpha}$ injection of the Ovsynch protocol $39 \pm 3 \mathrm{~d}$ after AI. Thereafter, nonpregnant cows from both programs received the second GnRH injection of Ovsynch $56 \mathrm{~h}$ after the $\mathrm{PGF}_{2 \alpha}$ injection and their next TAI within 16 to $20 \mathrm{~h}$ after the $\mathrm{GnRH}$ injection. The resulting IBI for cows inseminated after resynchronization of ovula- tion was 35 and $42 \mathrm{~d}$ for CT31 and RP39, respectively (Figure 1A).

The second comparison consisted of an early testing strategy using a CT $24 \pm 3$ d after AI (CT24), and a late testing program using TU at $32 \mathrm{~d}$ after AI (TU32; Figure 1B). For CT24, cows initiated the Ovsynch protocol $18 \pm 3 \mathrm{~d}$ after $\mathrm{AI}$ by receiving a $\mathrm{GnRH}$ injection, and cows diagnosed nonpregnant by the $\mathrm{CT}$ received the $\mathrm{PGF}_{2 \alpha}$ injection of the Ovsynch protocol $25 \pm 3 \mathrm{~d}$ after AI. In contrast, TU32 cows initiated the Ovsynch protocol $25 \pm 3$ d after AI by receiving a GnRH injection, and cows diagnosed nonpregnant using $\mathrm{TU}$ received the $\mathrm{PGF}_{2 \alpha}$ injection of the Ovsynch protocol $32 \pm 3 \mathrm{~d}$ after AI. Thereafter, nonpregnant cows from both programs received the second GnRH injection of Ovsynch $56 \mathrm{~h}$ after the $\mathrm{PGF}_{2 \alpha}$ injection and their next TAI within 16 to $20 \mathrm{~h}$ after the $\mathrm{GnRH}$ injection. The resulting IBI for cows inseminated after resynchronization of ovulation were 28 and $35 \mathrm{~d}$ for CT24 and TU32, respectively (Figure 1B).

Table 2. Baseline and range for parameters for 2 hypothetical scenarios using an early chemical pregnancy test compared with rectal palpation or transrectal ultrasound

\begin{tabular}{|c|c|c|c|c|c|c|}
\hline \multirow[b]{2}{*}{ Item } & \multicolumn{3}{|c|}{ CT31 vs. RP39 ${ }^{1}$} & \multicolumn{3}{|c|}{ CT24 vs. TU32 ${ }^{2}$} \\
\hline & Baseline & Minimum & Maximum & Baseline & Minimum & Maximum \\
\hline Sensitivity (\%) & 98 & 94 & 99 & 97 & 94 & 99 \\
\hline Specificity (\%) & 98 & 94 & 99 & 97 & 94 & 99 \\
\hline Pregnancy $\operatorname{loss}^{3}(\%)$ & 6.0 & 0 & 10 & 6.6 & 0 & 10 \\
\hline Questionable diagnosis (\%) & 3.3 & 0 & 10 & 8.5 & 0 & 10 \\
\hline $\mathrm{AIED}^{4}(\%)$ & 50 & 30 & 80 & 50 & 30 & 80 \\
\hline Cost of chemical test ${ }^{5}$ ( $\$ /$ test $)$ & 2.4 & 0.5 & 5.0 & 2.4 & 0.5 & 5.0 \\
\hline
\end{tabular}

${ }^{1}$ Early test performed using a chemical blood test at $31 \pm 3 \mathrm{~d}$ (CT31) resulted in an interbreeding interval for cows receiving a timed AI service of $35 \mathrm{~d}$, whereas for the other program nonpregnancy diagnosis was performed $8 \mathrm{~d}$ later by rectal palpation at $39 \pm 3 \mathrm{~d}$ (RP39) after AI, which resulted in an interbreeding interval of $42 \mathrm{~d}$.

${ }^{2}$ Early test performed using a chemical blood test at $24 \pm 3 \mathrm{~d}$ (CT24) resulted in an interbreeding interval of $28 \mathrm{~d}$, whereas late test performed by transrectal ultrasound at $32 \pm 3 \mathrm{~d}$ (TU32) resulted in an interbreeding interval of $35 \mathrm{~d}$.

${ }^{3}$ During the 8-d period between early and late pregnancy tests (CT31 vs. RP39 d and CT24 vs. TU32 d) based on Vasconcelos et al. (1997).

${ }^{4} \mathrm{AIED}=$ percentage of cows receiving AI after detection of estrus ether before first timed AI or between resynchronized timed AI services.

${ }^{5}$ First pregnancy test after AI. 


\section{Experiment 2: Modifications to the UW-DairyRepro\$ Decision Support Tool}

The UW-DairyRepro\$ decision support tool uses a Markov chain simulation model to determine the reproductive status of cows in a herd and hence the herd dynamics, as described in Giordano et al. (2011). The model sequentially estimates the percentage of cows eligible for breeding after the end of the VWP, the proportion of cows receiving AI, the percentage of cows becoming pregnant, and the percentage of cows not becoming pregnant at each service. In the first state after the end of the VWP, nonpregnant cows could move to the next state of being inseminated based on transition probabilities determined by the estrus detection rate for programs relying on visual detection of estrus or the predefined timing of AI for programs using synchronization. For reproductive management programs that combined both strategies (i.e., detection of estrus and synchronization), cows were inseminated after detection of estrus after the end of the VWP or a previous AI, whereas cows not detected in estrus completed the synchronization protocol to receive TAI.

Cows receiving AI moved to the pregnant state based on transition probabilities of CR defined by the reproductive program. Conversely, cows failing to conceive as well as cows not receiving an AI returned to the nonpregnant state and were eligible for the next AI. This probabilistic process was endowed by the Markov property that a next state depends solely on the current state and the transition probabilities: $P_{s}=$ $(C R)_{s} \times(S R)_{s}$, where $P_{s}$ is the proportion of pregnant cows in each service attempt, $s ; C R$ is the number of pregnant cows per number of inseminated cows; service rate $(\boldsymbol{S R})$ is the proportion of cows bred out from total number of breeding eligible after the end of the VWP, and $s$ is the AI service number. The proportion of cows inseminated in each service attempt was determined by $S R_{s}=B E_{s} \times B$, where $B E=$ proportion of breeding eligible cows and $B=$ proportion of bred cows. As defined by the reproductive program, breeding-eligible cows are those cows nonpregnant (open) after the previous AI service $\left[B E_{s}=\left(1-P_{s-1}\right)\right]$, whereas inseminated cows are those receiving an AI after either detection of estrus or completion of a synchronization protocol. The IBI for synchronized services is defined by the protocol. The percentage of cows not becoming pregnant after a series of reproductive services for a defined reproductive program (reproductive services to defined cut-off DIM) is the complement of the aggregated proportion of cows becoming pregnant, which is calculated as the cumulative sum of pregnancies until a defined DIM, that is,

$$
N P_{S}=1-\sum_{s=1}^{S} P_{s},
$$

where $N P$ is the proportion of nonpregnant cows after cut-off DIM after last AI service attempt. The number of services to a defined cut-off DIM depends on the reproductive program with the last reproductive event occurring when DIM $\leq$ defined cut-off DIM.

Additional parameters were introduced into the UW-DairyRepro\$ decision support tool to account for the reproductive and economic effects of the potential inaccuracy of an early nonpregnancy diagnosis using a CT. The parameters included were sensitivity (Se), specificity (Sp), questionable diagnosis (Qd), cost of the CT, and expected pregnancy loss. Sensitivity and Sp were defined using the test to which the CT was being compared with as a gold standard. In addition, pregnancy loss for this particular study was defined as the total percentage of cows that underwent pregnancy loss during the 8-d period between the early CT and the later RP or TU tests.

Sensitivity. This value multiplied the $\mathrm{CR}$ at each AI service: $P_{s}=(C R \times S e)_{s} \times(S R)_{s}$, because a test with $<100 \%$ Se will fail to detect a proportion of pregnant cows (false negative). These cows misdiagnosed as nonpregnant will lose their pregnancy when submitted to the TAI because they receive a $\mathrm{PGF}_{2 \alpha}$ injection as part of the resynchronization of ovulation protocol. Cows losing their pregnancy due to a false negative diagnosis were added to the nonpregnant group after each AI service attempt.

Specificity. This value was multiplied by the proportion of expected nonpregnant cows in each breeding period: $B E_{s}=\left(1-P_{s-1}\right) \times(S p)$, because a test with $<100 \%$ Sp will fail to detect a proportion of nonpregnant cows. These cows are misdiagnosed as pregnant (false positive). Therefore, the complement of the Sp (i.e., $100 \%$ - Sp) was used to account for the proportion of false pregnant cows. Because cows misdiagnosed as pregnant can only be re-inseminated if detected in estrus or when enrolled into the synchronization program after a nonpregnancy diagnosis at reconfirmation, these cows had a delayed re-insemination. This subgroup of cows was eligible for re-insemination at a detected estrus or received TAI after being diagnosed open at the pregnancy reconfirmation. Pregnancy reconfirmation was set at $28 \mathrm{~d}$ after the first pregnancy diagnosis.

Questionable Diagnosis. When the results of any pregnancy test are inconclusive, cows are assigned a Qd and retested after a certain number of days. For cows with a $\mathrm{Qd}$, retesting (recheck) was assumed to be performed 8 d later; hence, cows that were actu- 
ally nonpregnant had delayed rebreeding. These cows were not allowed to receive their next TAI after the inconclusive diagnosis and were eligible for AI after detection of estrus in the next cycle or received TAI after re-enrollment in the synchronization program. The total proportion of $\mathrm{Qd}$ was first split into a questionable pregnant diagnosis $(71.43 \%)$ and questionable nonpregnant diagnosis $(28.57 \%)$, according to Romano and Larson (2010). The model accounted for the cost of additional rechecks for cows with previous Qd, assuming same costs as the first test.

Pregnancy Loss. This value was added to the population of false pregnant cows in each breeding period because these cows, although correctly diagnosed as pregnant, will lose their pregnancy. Consequently, they can only be re-inseminated if they are either detected in estrus or found nonpregnant $28 \mathrm{~d}$ later at the time of their pregnancy reconfirmation. Because the effect of pregnancy loss on herd dynamics is similar to that of decreased Sp, 1 percentage point of increased pregnancy loss was equivalent to 1 percentage point of decreased Sp; therefore, no modifications of the model equations were required. When comparing CT31 and RP39, pregnancy loss from 31 to 39 d after TAI was set at $0.75 \%$ per day, based on Vasconcelos et al. (1997). When comparing CT24 and TU32, pregnancy loss from 24 to 28 d after TAI was set at $0.9 \%$ per day to account for a slightly greater pregnancy loss at earlier stages of gestation (no published data were available from 24 to $28 \mathrm{~d}$ after TAI) and $0.75 \%$ per day from 28 to $32 \mathrm{~d}$ after TAI, based on Vasconcelos et al. (1997).

Cost of the CT. This value was added to the cost of hormones, labor, and AI to the first and subsequent services, including estrus detection and TAI within the model structure. No modifications of the model were required.

Each parameter was divided into 4 levels from minimum to maximum (Table 2) and were used as inputs in the modified UW-DairyRepro\$ decision support tool. Simulations were run for all possible combinations of parameter values $\left(4^{6}=4,096\right.$ scenarios for each comparison). Results were then summarized in data sets and analyzed by multiple regression analysis using PROC REG of SAS (SAS Institute Inc., Cary, NC) and breakeven analyses.

\section{RESULTS}

\section{Experiment 1: Effect of IBI on the Value of Reproductive Programs}

The NPV (\$/cow per year) for all reproductive programs simulated is presented in Figure 2. Programs with a shorter IBI had greater NPV at all levels of
AIED. The NPV also showed a positive trend with increasing percentage of cows inseminated after detection of estrus from 30 to $80 \%$. The effect of adding cows inseminated after ED was greater for programs with a longer IBI compared with those programs with a shorter IBI, which presented smaller gains when increasing the percentage of cows inseminated after ED.

\section{Experiment 2: Effect of CT Accuracy on Survival Curves to Pregnancy}

Figure 3 depicts the implications on survival curves to pregnancy of different levels of accuracy of a CT after simulating extreme conditions of Se, Sp, Qd, and expected pregnancy loss for the first pregnancy diagnosis after AI. The RP39 program, with a resulting IBI of 42 $\mathrm{d}$ for TAI services, was used as baseline for comparison with the CT31 program, with an IBI of $35 \mathrm{~d}$ for TAI services. The programs selected for presentation of data in Figure 3 include those with $50 \%$ of cows AI after detection of estrus as described in Table 1. By the end of the breeding period, the proportion of pregnant cows was $89.0 \%$ when using RP. In contrast, the percentage of pregnant cows was 92.1 and $88.3 \%$ when using a CT 8 d earlier under extreme low and high conditions of Se, Sp, Qd, and pregnancy loss, respectively. In the

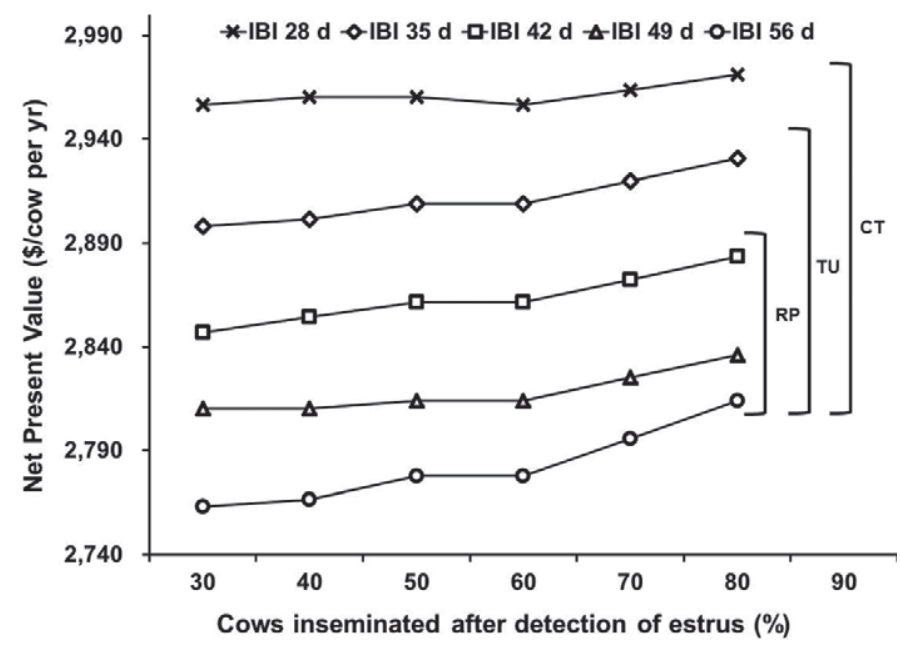

Figure 2. Effect of interbreeding interval (IBI) on the net present value for simulated reproductive management programs combining detection of estrus with the Presynch-Ovsynch protocol for first timed AI (TAI) service and the Ovsynch protocol for resynchronization of ovulation for second and greater TAI services. The IBI varied from 28 to $56 \mathrm{~d}$. For each IBI, the percentage of cows receiving AI after detection of estrus before first TAI and between resynchronized TAI ranged from 30 to $80 \%$ in 10-percentage-point increments. Brackets indicate the set of interbreeding intervals using Ovsynch for resynchronization of ovulation and TAI that can be achieved when using rectal palpation $(\mathrm{RP})$, transrectal ultrasound (TU), and a chemical test (CT) for nonpregnancy diagnosis. The program with 28 -d IBI is not currently possible with commercially available $\mathrm{CT}$ but might be in the future. 
first case, $92.1 \%$ of the cows were pregnant by the end of the breeding period when Se and Sp were 99\%, Qd $0 \%$, and pregnancy loss $0 \%$. Conversely, $88.3 \%$ of the cows were pregnant by the end of the breeding period when Se and Sp were 94\%, Qd 10\%, and pregnancy loss $10 \%$. Similar results were observed for the comparison between CT24 and TU32 (data not shown).

\section{Experiment 2: Sensitivity Analysis}

The NPV for 4,096 scenarios (4 levels for each of 6 factors) comparing programs CT31 and RP39 and a similar number of scenarios comparing the NPV of programs CT24 and TU32 were simulated and their NPV calculated. Results for CT31 compared with RP39 are shown in Figure 4.

For the comparison of CT31 and RP39, the economic value of CT31 was affected (in order) by (1) Se, (2) Sp, (3) pregnancy loss, (4) proportion of Qd, (5) percentage AIED, and (6) the cost of CT, as shown in Figure 4. The greater or lower NPV of CT31 compared with RP39 was represented by the difference between the NPV of CT31 (dots) and that of RP39 (dashed line). Therefore, dots above the dashed line for a specific combination of factors under consideration (Se, Sp, Qd, pregnancy loss, cost of the test and percentage of cows

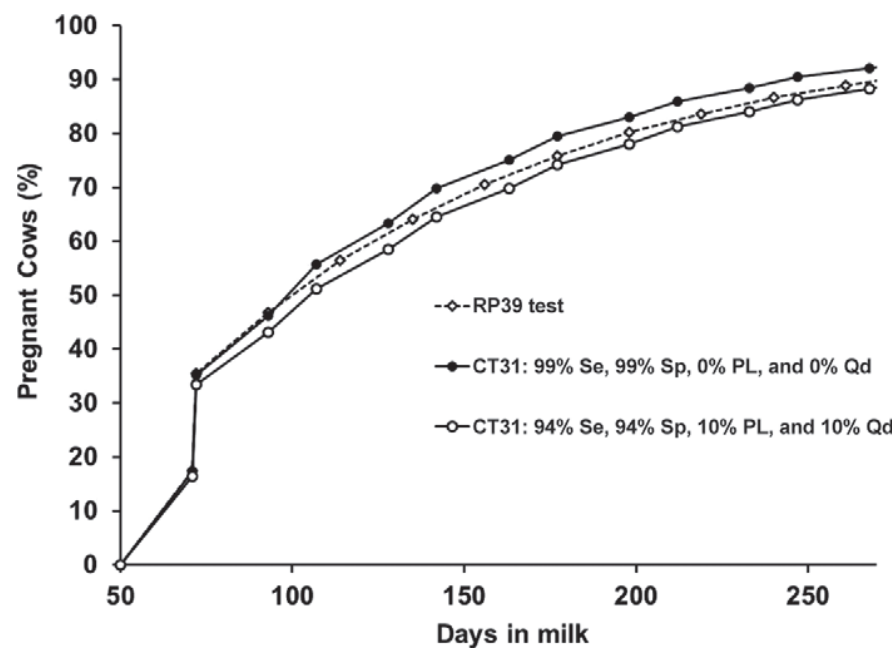

Figure 3. Effect of the accuracy of a chemical test (CT) on the reproductive performance of lactating dairy cows under hypothetical conditions of sensitivity (Se), specificity ( $\mathrm{Sp}$ ), percentage of questionable diagnosis (Qd), and pregnancy loss (PL). Survival curves to pregnancy for 3 scenarios representing reproductive management programs combining detection of estrus (percentage of cows AI after detection of estrus was set at 50\%) with timed AI (TAI) after synchronization of ovulation with the Presynch-Ovsynch protocol for first TAI and the Ovsynch protocol for second and subsequent TAI. A program using rectal palpation (RP39) as the method for nonpregnancy diagnosis 39 $\pm 3 \mathrm{~d}$ after $\mathrm{AI}$ and resulting in a 42-d interbreeding interval for cows receiving TAI was compared with 2 scenarios in which CT was used for determination of pregnancy status $31 \pm 3 \mathrm{~d}$ after AI (CT31) under hypothetical conditions of Se, Sp, Qd, and pregnancy loss.
AI after detection of estrus) represent a gain in NPV if CT31 (vs. RP39) is performed. Regardless of a different magnitude in NPV differences between the hypothetical scenarios simulated in the sensitivity analysis, a similar situation was observed when comparing CT24 and TU32 (data not shown).

Because each of the 6 factors was divided into 4 sections (Table 2), we can assess general trends to elucidate interactions between the main factors compared by examining Figure 4 . The percentage AIED splits the figure into 4 distinguishable sections from low $(30 \%)$ to high $(80 \%)$ percentage of cows AI after detection of estrus. Within each AIED block, 4 sections are distinguishable, indicating Se from high (99\%) to low (94\%) (Figure 4; section A). The Se of the CT was the most important factor determining its value, based on the large difference between the first and fourth block within section A. When Se was high, most outcomes were above the horizontal dashed line (positive values). Conversely, when Se was low (fourth block), only a few outcomes were above the horizontal dashed line, with most appearing below the line. Data in Figure 4 are divided into blocks that represent quartiles for the values of each factor run. Within each Se block, 4 distinguishable blocks indicating Sp from high (99\%) to low (94\%; Figure 4, section B). Specificity of the CT is the second factor of importance in determining its value. The effect of Sp can be assessed by observing the difference between the upper and lower block of outcomes in each Sp block. Similarly, within the Sp blocks, 4 blocks showed the effect of pregnancy loss from low (0\%) to high (10\%; Figure 4, section C). Higher NPV values were observed with lower pregnancy loss.

Magnification of the area for each pregnancy loss level reveals the effect of the percentage of $\mathrm{Qd}$ and the cost of the CT (Figure 4, section D). Comparing the 4 horizontal blocks (from left to right) in the detailed area, the relative effect of varying the percentage of $\mathrm{Qd}$ from low $(0 \%)$ to high $(10 \%)$ can be assessed. Finally, by comparing the outcomes with each of the 4 vertical blocks, the relative effect of the cost of the CT from low (\$0.5) to high $(\$ 5.0)$ can be appreciated (Figure 4, section E). Compared with other factors, the cost of the CT was the least important factor affecting the value of the earlier CT.

\section{Experiment 2: Regression Analysis to Quantify the Effect of the Earlier CT}

The NPV for the 4,096 scenarios previously generated to compare programs CT31 and RP39 and programs CT24 and TU32 were analyzed by multiple regression to determine the quantitative effect of each factor on the value of the CT (Table 3). Regression coefficients 


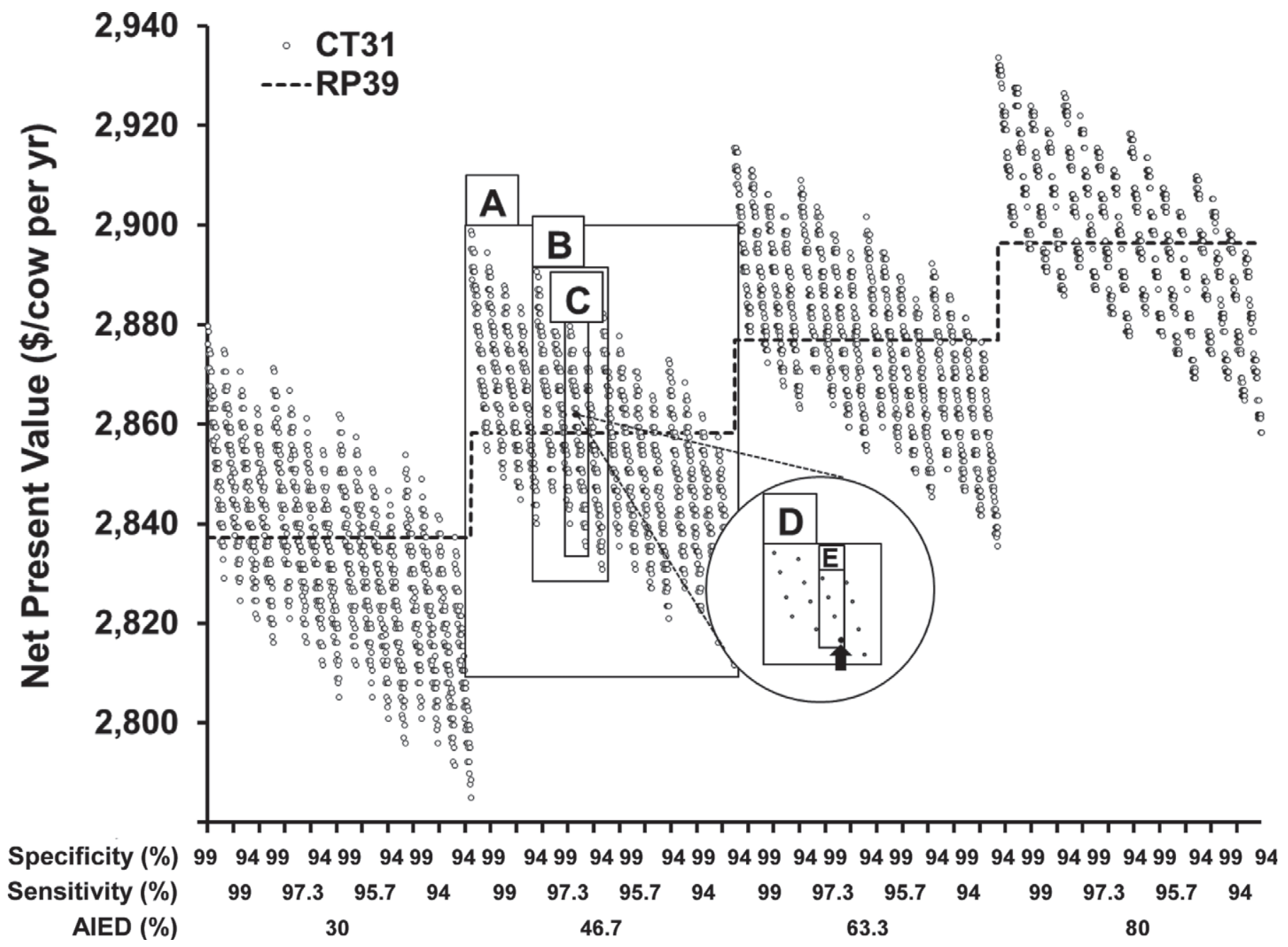

Figure 4. The value of early nonpregnancy diagnosis with a chemical test $31 \pm 3 \mathrm{~d}$ (CT31) after AI compared with rectal palpation test $39 \pm 3 \mathrm{~d}$ (RP39) after AI according to 4 levels of 6 different factors: (1) percentage of cows AI after detection of estrus (AIED; 30 to $80 \%$ ), (2) sensitivity (99 to $94 \%$, section A), (3) specificity (99 to $94 \%$, section B), (4) pregnancy loss (0 to 10\%, section C), (5) questionable diagnosis (0 to $10 \%$, section D; not included in x-axis), and (6) cost of CT (\$0.5 to $\$ 5$, section E; not included in x-axis). The magnitude of the variation in observations for questionable diagnosis and cost of the CT was minimal, requiring magnification of the last visible dots (sections D and E) to appreciate the differences. The observations (dots) included 4,096 potential combinations of the 6 factors included in the analysis. Each factor was divided in 4 blocks corresponding to each quartile for the range of values considered (e.g., for cows AI after detection of estrus the range was 30 to $80 \%$; therefore, the quartiles were 30,46.7,63.3, and 80\%). As an example, the highlighted dot (black arrow) represents the net present value of CT31 when AIED is $46.7 \%$, and the following values for the CT: sensitivity of $97.3 \%$, specificity of $95.7 \%$, pregnancy loss of $3.3 \%$, questionable diagnosis $6.7 \%$, and cost of the CT $\$ 5$. Outcomes above the dashed line represent situations in which positive values for the chemical test were found compared with the later rectal palpation.

for each factor under consideration in the analysis were used to approximate the difference between programs in their NPV (difference between the earlier CT and the later RP or TU test) under different conditions. The added value of the earlier CT under ideal hypothetical conditions of high test accuracy (implying 99\% Se, $99 \%$ Sp, and 0\% Qd), and assuming no pregnancy loss, for a program with $50 \%$ of cows inseminated after detection of estrus and a test cost of $\$ 2.40$ would be $\$ 36.78$ and $\$ 43.33 /$ cow per year when comparing CT31 to RP39 and CT24 to TU32, respectively. Nevertheless, because of a certain degree of inaccuracy expected for the CT and due to naturally occurring pregnancy loss, more realistic conditions were compared. Considering a situation of $98 \%$ Se, $98 \% \mathrm{Sp}, 3.3 \%$ Qd for CT31, and $6.0 \%$ pregnancy loss, the added value of the CT was $\$ 8.77 /$ cow per year. For the more aggressive scenario with CT24 versus TU32, considering $97 \%$ Se, $97 \%$ Sp, $8.5 \% \mathrm{Qd}$, and $6.6 \%$ pregnancy loss, the added value of the earlier CT was $\$ 9.66 /$ cow per year. 
Table 3. Regression coefficients and quantitative effects of the 6 factors evaluated on the difference between the value of the early chemical test (net present value difference; $\$ /$ cow per year) and rectal palpation or transrectal ultrasound

\begin{tabular}{|c|c|c|c|c|c|c|}
\hline \multirow[b]{2}{*}{ Item } & \multicolumn{3}{|c|}{ CT31 vs. RP39 ${ }^{1}$} & \multicolumn{3}{|c|}{ CT24 vs. TU32 ${ }^{2}$} \\
\hline & $\begin{array}{l}\text { Regression } \\
\text { coefficient }\end{array}$ & $\begin{array}{l}\text { Quantitative } \\
\text { effect }^{3}\end{array}$ & $\begin{array}{l}\text { Relative effect to } \\
\text { sensitivity }\end{array}$ & $\begin{array}{l}\text { Regression } \\
\text { coefficient }\end{array}$ & $\begin{array}{l}\text { Quantitative } \\
\text { effect }\end{array}$ & $\begin{array}{l}\text { Relative effect } \\
\text { to sensitivity }\end{array}$ \\
\hline Constant & -795.4 & & & -637.7 & & \\
\hline Sensitivity (\%) & 534.5 & +5.3 & - & 450.3 & +4.5 & - \\
\hline Specificity (\%) & 305.4 & +3.1 & 1.8 & 253.4 & +2.5 & 1.8 \\
\hline Pregnancy loss (\%) & -305.4 & -3.1 & -1.8 & -253.4 & -2.5 & -1.8 \\
\hline Cost of chemical pregnancy test $(\$)$ & -1.75 & -0.0175 & -305.8 & -1.92 & -0.0192 & -235.1 \\
\hline
\end{tabular}

${ }^{1}$ Early test performed using a chemical blood test at $31 \pm 3 \mathrm{~d}$ (CT31) resulted in an interbreeding interval for cows receiving a timed AI service of $35 \mathrm{~d}$, whereas for the other program nonpregnancy diagnosis was performed $8 \mathrm{~d}$ later by rectal palpation at $39 \pm 3 \mathrm{~d}$ (RP39) after AI, which resulted in an interbreeding interval of $42 \mathrm{~d}$.

${ }^{2}$ Early test performed using a chemical blood test at $24 \pm 3 \mathrm{~d}$ (CT24) resulted in an interbreeding interval of 28 d, whereas late test performed by transrectal ultrasound at $32 \pm 3 \mathrm{~d}$ (TU32) resulted in an interbreeding interval of $35 \mathrm{~d}$.

${ }^{3}$ Expressed as $\$$ per 1-percentage-point increase or $\$$ per $\$ 0.1$ increase.

${ }^{4}$ Regression coefficient of sensitivity divided by regression coefficient of analyzed factor.

\section{Experiment 2: Breakeven Analysis}

A breakeven (the point where the economic benefit or added value of one program over another becomes zero) analysis was performed using the regression coefficients from Table 3 and baseline values for the CT described in Table 2. For CT31 versus RP39, an economic breakeven occurred when Se was $96.4 \%$, Sp was $95.1 \%$, and the pregnancy loss was $8.9 \%$, when all other parameters were as specified in Table 2. For CT24 versus TU32, an economic breakeven was reached if Se was $94.9 \%$, Sp was $93.2 \%$, and the pregnancy loss was $10.5 \%$, when all other parameters were as specified in Table 2.

\section{DISCUSSION}

As expected, the modeled scenarios clearly demonstrated that reducing the interval between 2 successive TAI services resulted in greater economic net returns. Because the CR of all AI services remained unchanged for the different IBI simulated, it was not surprising that shorter IBI resulted in greater NPV. A shorter IBI between TAI services affects herd dynamics by generating more pregnancies in a shorter period after the end of the VWP. Therefore, any method for nonpregnancy diagnosis that allows the implementation of reproductive management programs that reduce the interval between 2 successive TAI services without affecting fertility will improve the profitability of the farm. Thus, use of CT available on the market today might be beneficial compared with RP because CT can be performed earlier after AI.

Another important observation was that increasing the percentage of cows receiving AI after detection of estrus always increased the NPV for all programs.
This occurred because the CR of AIED for second and subsequent AI services was $35 \%$, which represents an improvement of either 5 or 7 percentage points compared with TAI. In addition, as the percentage of AIED increased, the NPV of programs continued to increase because more cows had a 22-d IBI compared with the longer IBI for cows receiving TAI. Similar observations were reported by Giordano et al. (2011) when adding estrus detection to a 100\% TAI D32 Resynch program with $30 \% \mathrm{CR}$ across all resynchronized AI services. Contrasting results, however, are observed when the fertility (CR) of AI services after detection of estrus is lower than that observed for cows receiving TAI (Giordano et al., 2012a).

The greater benefit of increasing the percentage of AIED in programs with longer IBI (steeper increase in NPV line) clearly reflected and supported this notion. The greatest benefits were observed when a significant percentage of cows received AI at estrus compared with receiving TAI at a longer IBI.

For simplification of simulation and interpretation of results, a major assumption of this analysis was that whatever test was being used resulted in $100 \%$ accuracy to detect nonpregnant and pregnant cows, had the same cost of application, and occurred in the absence of pregnancy loss. Because this may not reflect the results observed in commercial operations, the effects of inaccurate diagnosis, variable costs, and pregnancy loss were addressed in experiment 2.

In general, the results of experiment 2 were consistent with those reported by Ferguson and Galligan (2011) and others (Oltenacu et al., 1990; Pitcher and Galligan 1990; Galligan et al., 2009). The order and direction of the effect of changing the values for the factors evaluated in this experiment for the CT (Se, Sp, Qd, CT cost, 
and pregnancy loss) were, for the most part, highly consistent with these earlier studies. Regarding the percentage of cows receiving AI after detection of estrus, we found both similar and, to some extent, contrasting results. When contrasting our results with those of others, it is important to emphasize that the framework used in the present study was substantially different. Most other studies (Oltenacu et al., 1990; Pitcher and Galligan 1990; Galligan et al., 2009) used different variations of decision-tree analysis, in which the accuracy of the pregnancy test was either rewarded or punished according to probabilistic outcomes. Conversely, in the present study, we used a comprehensive approach that considered the effect of the $\mathrm{CT}$ on the dynamics of the whole lactating herd. Moreover, whereas in previous studies the effect of using different pregnancy tests was assessed at the same number of days after AI, we also included in our analysis the added value of earlier testing through technologies readily available today for nonpregnancy diagnosis around $30 \mathrm{~d}$ after AI (ultrasound and blood chemical tests) and the use of new technologies potentially available in the future (blood testing around $20 \mathrm{~d}$ after $\mathrm{AI}$ ).

The Se of the CT was the most important test characteristic and had the greatest effect on its relative value. Indeed, the effect of Se on the total value was 1.8 times greater than for the Sp. In contrast, Ferguson and Galligan (2011) reported a 4-times-greater magnitude for Se than for Sp. Oltenacu et al. (1990) reported that the error rate for an earlier pregnancy test needs to be $\leq 3 \%$ to have a positive value. Ferguson and Galligan (2011) reported that the test value would become negative if the Se is less than $90 \%$. To obtain a positive value for the $\mathrm{CT}$, we determined that Se should be approximately greater than $96 \%$ when the CT is used at $31 \pm 3 \mathrm{~d}$ and greater than $94 \%$ when used $24 \pm 3$ $\mathrm{d}$ after AI. Certainly, some of the differences between studies are due to the different frameworks used for analysis. One important difference is that we defined Se as a relative value of the pregnancy test used for the later diagnosis, which was considered the gold standard (having 100\% accuracy). Because no test has 100\% accuracy, the actual value of the $\mathrm{CT}$ in the present study may have been slightly underestimated.

A test with low Se results in the misdiagnosis of truly pregnant cows as nonpregnant. When these misdiagnosed cows continue into the synchronization protocol, iatrogenic pregnancy loss will occur after they receive a $\mathrm{PGF}_{2 \alpha}$ injection. Induced pregnancy losses are certainly expensive; however, they may not be as expensive as previously reported. Our model accounted internally for the value of these pregnancy losses (Giordano et al., 2011), whereas this is an external input in the decisiontree approach. We speculate that the value associ- ated with induced early pregnancy loss may have been overestimated by others. For example, Galligan et al. (2009) used $\$ 300$ per pregnancy loss, whereas Ferguson and Galligan (2011) used only $\$ 46$, recognizing that a lower value should be used because cows experiencing induced early pregnancy loss are re-inseminated within $10 \mathrm{~d}$ of the misdiagnosis.

A test with low Sp leads to the misclassification of cows as pregnant when they are truly nonpregnant. Consequently, instead of completing the resynchronization protocol and receiving their next TAI, these cows with a false pregnant diagnosis will not be re-inseminated until they are either detected in estrus or diagnosed not pregnant at the pregnancy reconfirmation. In our approach, another group of cows that followed the same dynamics were those experiencing pregnancy loss. These cows, however, were correctly classified pregnant by the test and subsequently lost their pregnancies, thereby resulting in delayed rebreeding when they either receive AI after ED or are diagnosed not pregnant at the next pregnancy reconfirmation. The value lost by cows experiencing pregnancy loss was similar to that of cows affected by the low Sp of the test being used. The relatively short interval $(28 \mathrm{~d}$ ) between the first and subsequent pregnancy diagnosis (recheck) used in this study may have attenuated the effect of both low Sp and pregnancy loss on the overall economic value of the reproductive programs. Greater intervals between pregnancy tests are expected to have a greater impact of the economic value of any diagnostic test.

In agreement with Ferguson and Galligan (2011), Qd had less of a negative effect than Se and Sp in the present study (Table 3). This was because, at least in part, cows with a Qd were re-examined within $7 \mathrm{~d}$ and if nonpregnant were promptly reassigned to the TAI program. Based on these observations, a Qd (or recheck) followed by retesting within $7 \mathrm{~d}$ after the initial test is preferable to a misdiagnosis.

The effect of the percentage of cows receiving AI after detection of estrus was the second to last factor influencing the value of the $\mathrm{CT}$, in contrast to the observations of Ferguson and Galligan (2011), who reported that percentage AIED had a greater influence on the value of a pregnancy test. This difference could have occurred because only highly aggressive reproductive programs combining TAI with estrus detection (more likely to implement a CT) were included in the present study. For this type of program, the effect of changing the percentage AIED may not be as dramatic as for programs that use $100 \%$ ED for insemination of cows, because cows not detected in estrus receive a TAI. Overall, the effect of increasing the percentage AIED on the final NPV of a program was positive under all circumstances. It was interesting, however, that 
increasing the percentage AIED decreased the specific value of the CT for comparison between programs with an IBI of 28 versus $35 \mathrm{~d}$, whereas an opposite trend was observed when comparing the programs with an IBI of 35 versus $42 \mathrm{~d}$. In the latter case, the difference in the expected value of the $\mathrm{CT}$ was greater at higher percentage AIED. This likely occurred because for longer IBI ( 35 vs. 42 d compared with 28 vs. 35 d), the effect of AIED increases.

Finally, consistent with Ferguson and Galligan (2011), the cost of the CT was not an important factor for determining the overall value of the test. In our analysis, the value of the conventional ( $\mathrm{TU}$ or $\mathrm{RP}$ ) tests was fixed at $\$ 2$ per cow, whereas the CT cost was allowed to change. For each $\$ 0.1$ increase in the CT cost, the value of the CT decreased by $\$ 0.18$ to $\$ 0.19$.

Even though we used a comprehensive approach that took into account the herd reproductive dynamics for this economic analysis of different pregnancy testing methods, other factors of importance in the value of a pregnancy test were beyond the scope of this study. For example, the involvement of a veterinarian in the reproductive management program may provide valuable information beyond a simple pregnancy diagnosis. Additional information, such as ovarian status, abnormalities of the reproductive tract, or abnormalities of the pelvic and abdominal cavity, may add value not taken into account by our analyses but should be accounted for during the decision-making process.

\section{CONCLUSIONS}

The major effect of using a chemical test for pregnancy testing in lactating dairy cows was the potential of shortening the IBI, with a consequent increase in herd profitability. When we included the potential inaccuracy of the chemical test in the analysis, smaller economic differences were observed for hypothetical scenarios performing the chemical test versus rectal palpation or transrectal ultrasound $8 \mathrm{~d}$ later. Rather than focusing on the value of the pregnancy test alone, the effect of using different testing methods on the outcome of the reproductive program applied at the farm should be considered. Although the results presented herein would not apply to every farm and reproductive management program, these scenarios are important to demonstrate a solid framework that could be perfected to perform economic assessments of using different testing methods as reproductive management programs for dairy cattle continue to evolve.

\section{ACKNOWLEDGMENTS}

This project was supported by Agriculture and Food Research Initiative Competitive Grant no. 2010-85122-
20612 from the USDA National Institute of Food and Agriculture (Washington, DC). The research was also supported by Hatch project WIS01171 to P.M.F and Hatch project WIS01577 to V.E.C.

\section{REFERENCES}

Branen, J. R., J. O. Giordano, C. Passavant, J. M. Howard, P. M. Fricke, and R. G. Sasser. 2011. Detection of early pregnancy and embryonic loss in dairy cows using BioPRYN and a NEW PSPBbased ELISA. J. Dairy Sci. 94(E-Suppl. 1):25. (Abstr.)

Chebel, R. C., M. J. Al-Hassan, P. M. Fricke, J. E. Santos, J. R. Lima, C. A. Martel, J. S. Stevenson, R. Garcia, and R. L. Ax. 2010. Supplementation of progesterone via controlled internal drug release inserts during ovulation synchronization protocols in lactating dairy cows. J. Dairy Sci. 93:922-931.

Chebel, R. C., and J. E. Santos. 2010. Effect of inseminating cows in estrus following a presynchronization protocol on reproductive and lactation performances. J. Dairy Sci. 93:4632-4643.

Ferguson, J. D., and D. T. Galligan. 2011. The value of pregnancy diagnosis - A revisit to an old art. 2011 Theriogenology Annu. Conf. Symp., Milwaukee, WI. Society of Theriogenology, Montgomery, AL.

Fricke, P. M., D. Z. Caraviello, K. A. Weigel, and M. L. Welle. 2003. Fertility of dairy cows after resynchronization of ovulation at three intervals following first timed insemination. J. Dairy Sci. $86: 3941-3950$.

Galligan, D. T., J. Ferguson, R. Munson, D. Remsburg, and A. Skidmore. 2009. Economic concepts regarding early pregnancy testing. Pages 48-53 in Proc. Am. Assoc. Bovine Pract., Omaha, NE. Am. Assoc. Bovine Pract., Auburn, AL.

Gifford, C. A., K. Racicot, D. S. Clark, K. J. Austin, T. R. Hansen, M. C. Lucy, C. J. Davies, and T. L. Ott. 2007. Regulation of interferon-stimulated genes in peripheral blood leukocytes in pregnant and bred, nonpregnant dairy cows. J. Dairy Sci. 90:274-280.

Giordano, J. O., P. M. Fricke, M. C. Wiltbank, and V. E. Cabrera. 2011. An economic decision-making support system for selection of reproductive management programs on dairy farms. J. Dairy Sci. 94:6216-6232.

Giordano, J. O., P. M. Fricke, M. C. Wiltbank, and V. E. Cabrera. 2012a. A daily herd Markov-chain model to study the reproductive and economic impact of reproductive programs combining timed artificial insemination and estrus detection. J. Dairy Sci. 95:5442-5460.

Giordano, J. O., M. C. Wiltbank, J. N. Guenther, R. Pawlisch, S. Bas, A. P. Cunha, and P. M. Fricke. 2012b. Increased fertility in lactating dairy cows resynchronized with Double-Ovsynch compared with Ovsynch initiated $32 \mathrm{~d}$ after timed artificial insemination. J. Dairy Sci. 95:639-653.

Green, J. A., T. E. Parks, M. P. Avalle, B. P. Telugu, A. L. McLain, A. J. Peterson, W. McMillan, N. Mathialagan, R. R. Hook, S. Xie, and R. M. Roberts. 2005. The establishment of an ELISA for the detection of pregnancy-associated glycoproteins (PAGs) in the serum of pregnant cows and heifers. Theriogenology 63:1481-1503.

Green, J. C., E. M. Newsom, and M. C. Lucy. 2011. Incorporation of a rapid pregnancy-associated glycoprotein ELISA into a CIDROvsynch resynchronization program for a 28 day re-insemination interval. Theriogenology 75:320-328.

Green, J. C., C. S. Okamura, S. E. Poock, and M. C. Lucy. 2010. Measurement of interferon-tau (IFN- $\tau$ ) stimulated gene expression in blood leukocytes for pregnancy diagnosis within 18-20 d after insemination in dairy cattle. Anim. Reprod. Sci. 121:24-33.

Green, J. C., D. H. Volkmann, S. E. Poock, M. F. McGrath, M. Ehrhardt, A. E. Moseley, and M. C. Lucy. 2009. Technical note: A rapid enzyme-linked immunosorbent assay blood test for pregnancy in dairy and beef cattle. J. Dairy Sci. 92:3819-3824.

Gumen, A., A. Keskin, G. Yilmazbas-Mecitoglu, E. Karakaya, A. Alkan, H. Okut, and A. Wiltbank. 2012. Effect of presynchronization strategy prior to Ovsynch on fertility at first service in lactating dairy cows. Theriogenology 78:1830-1838. 
Moreira, F., C. Orlandi, C. A. Risco, R. Mattos, F. Lopes, and W. W. Thatcher. 2001. Effects of presynchronization and bovine somatotropin on pregnancy rates to a timed artificial insemination protocol in lactating dairy cows. J. Dairy Sci. 84:1646-1659.

Oliveira, J. F., L. E. Henkes, R. L. Ashley, S. H. Purcell, N. P. Smirnova, D. N. Veeramachaneni, R. V. Anthony, and T. R. Hansen. 2008. Expression of interferon (IFN)-stimulated genes in extrauterine tissues during early pregnancy in sheep is the consequence of endocrine IFN-tau release from the uterine vein. Endocrinology 149:1252-1259.

Oltenacu, P. A., J. D. Ferguson, and A. J. Lednor. 1990. Economic evaluation of pregnancy diagnosis in dairy cattle: A decision analysis approach. J. Dairy Sci. 73:2826-2831.

Pitcher, P. M., and D. T. Galligan. 1990. Decision analysis and economic evaluation of the use of the rapid milk progesterone assay for early detection of pregnancy status of cows. J. Am. Vet. Med. Assoc. 197:1586-1590.

Pursley, J. R., M. O. Mee, and M. C. Wiltbank. 1995. Synchronization of ovulation in dairy cows using PGF2alpha and GnRH. Theriogenology 44:915-923.

Romano, J. E., and J. E. Larson. 2010. Accuracy of pregnancy specific protein-B test for early pregnancy diagnosis in dairy cattle. Theriogenology 74:932-939.

Santos, J. E., W. W. Thatcher, R. C. Chebel, R. L. Cerri, and K. N. Galvao. 2004. The effect of embryonic death rates in cattle on the efficacy of estrus synchronization programs. Anim. Reprod. Sci. 82-83:513-535.

Sasser, R. G., C. A. Ruder, K. A. Ivani, J. E. Butler, and W. C. Hamilton. 1986. Detection of pregnancy by radioimmunoassay of a novel pregnancy-specific protein in serum of cows and a profile of serum concentrations during gestation. Biol. Reprod. 35:936-942.

Silva, E., R. A. Sterry, D. Kolb, N. Mathialagan, M. F. McGrath, J. M. Ballam, and P. M. Fricke. 2007. Accuracy of a pregnancyassociated glycoprotein ELISA to determine pregnancy status of lactating dairy cows twenty-seven days after timed artificial insemination. J. Dairy Sci. 90:4612-4622.

Silva, E., R. A. Sterry, D. Kolb, N. Mathialagan, M. F. McGrath, J. M. Ballam, and P. M. Fricke. 2009. Effect of interval to resynchronization of ovulation on fertility of lactating Holstein cows when using transrectal ultrasonography or a pregnancy-associated glycoprotein enzyme-linked immunosorbent assay to diagnose pregnancy status. J. Dairy Sci. 92:3643-3650.

Stevenson, J. L., J. C. Dalton, T. L. Ott, K. E. Racicot, and R. C. Chebel. 2007. Correlation between reproductive status and steadystate messenger ribonucleic acid levels of the Myxovirusresistance gene, MX2, in peripheral blood leukocytes of dairy heifers. J. Anim. Sci. 85:2163-2172.

Vasconcelos, J. L. M., R. W. Silcox, J. A. Lacerda, J. R. Pursley, and M. C. Wiltbank. 1997. Pregnancy rate, pregnancy loss, and response to estrous stress after AI at two different times from ovulation in dairy cows. Biol. Reprod. 56(Suppl. 1):140. (Abstr.)

Zoli, A. P., L. A. Guilbault, P. Delahaut, W. B. Ortiz, and J. F. Beckers. 1992. Radioimmunoassay of a bovine pregnancy-associated glycoprotein in serum: Its application for pregnancy diagnosis. Biol. Reprod. 46:83-92. 